\title{
Transcriptome and expression profiling analysis revealed changes of multiple signaling pathways involved in immunity in the large yellow croaker during Aeromonas hydrophila infection
}

Yinnan $\mathrm{Mu}^{1+}$, Feng Ding ${ }^{2+}$, Peng $\mathrm{Cui}^{2}$, Jingqun $\mathrm{Ao}^{1}$, Songnian $\mathrm{Hu}^{2^{*}}$, Xinhua Chen ${ }^{1 *}$

\begin{abstract}
Background: The large yellow croaker (Pseudosciaena crocea) is an economically important marine fish in China suffering from severe outbreaks of infectious disease caused by marine bacteria such as Aeromonas hydrophila (A. hydrophila), resulting in great economic losses. However, the mechanisms involved in the immune response of this fish to bacterial infection are not fully understood. To understand the molecular mechanisms underlying the immune response to such pathogenic bacteria, we used high-throughput deep sequencing technology to investigate the transcriptome and comparative expression profiles of the large yellow croaker infected with A. hydrophila.
\end{abstract}

Results: A total of 13,611,340 reads were obtained and assembled into 26,313 scaffolds in transcriptional responses of the A. hydrophila-infected large yellow croaker. Via annotation to the NCBI database, we obtained 8216 identified unigenes. In total, 5590 (68\%) unigenes were classified into Gene Ontology, and 3094 unigenes were found in 20 KEGG categories. These genes included representatives from almost all functional categories. By using Solexa/lllumina's DeepSAGE, 1996 differentially expressed genes ( $P$ value $<0.05$ ) were detected in comparative analysis of the expression profiles between $A$. hydrophila-infected fish and control fish, including 727 remarkably upregulated genes and 489 remarkably downregulated genes. Dramatic differences were observed in genes involved in the inflammatory response. Bacterial infection affected the gene expression of many components of signaling cascades, including the Toll-like receptor, JAK-STAT, and MAPK pathways. Genes encoding factors involved in T cell receptor (TCR) signaling were also revealed to be regulated by infection in these fish.

Conclusion: Based on our results, we conclude that the inflammatory response may play an important role in the early stages of infection. The signaling cascades such as the Toll-like receptor, JAK-STAT, and MAPK pathways are regulated by $A$. hydrophila infection. Interestingly, genes encoding factors involved in TCR signaling were revealed to be downregulated by infection, indicating that TCR signaling was suppressed at this early period. These results revealed changes of multiple signaling pathways involved in immunity during A. hydrophila infection, which will facilitate our comprehensive understanding of the mechanisms involved in the immune response to bacterial infection in the large yellow croaker.

\footnotetext{
* Correspondence: husn@big.ac.cn; chenxinh@tom.com

+ Contributed equally

${ }^{1}$ Key Laboratory of Marine Biogenetic Resources, Third Institute of

Oceanography, State Oceanic Administration, Xiamen 361005, China

${ }^{2}$ The CAS Key Laboratory of Genome Sciences and Information, Beijing

Institute of Genomics, Chinese Academy of Sciences, Beijing 100029, China

Full list of author information is available at the end of the article
} 


\section{Background}

The large yellow croaker (Pseudosciaena crocea) is an economically important marine fish in China, with an annual yield that exceeds any other single netcagefarmed marine species. However, recent rapid development of the large yellow croaker farming industry has led to increasingly severe outbreaks of infectious disease caused by marine bacteria such as Aeromonas hydrophila (A. hydrophila), resulting in great economic losses [1]. However, little is known about the molecular mechanisms underlying the immune response to such pathogenic bacteria in this fish species, thereby hindering the establishment of effective measures in disease control [2].

Cellular identity and function are determined by the transcriptome or the complete repertoire of expressed RNA transcripts. Transcriptome profiling is a powerful method for assessing the relative importance of gene products in any chosen cell, tissue, organism, or condition. During the last few years, several methods have been used to study the fish transcriptome, including ESTs in channel catfish [3], Atlantic salmon [4], and orange-spotted grouper [5], as well as microarrays in adult zebrafish [6], rainbow trout [7], blue catfish [8], medaka, and Xiphophorus maculates [9]. However, microarrays are limited by background and crosshybridization problems and only measure the relative abundance of transcripts. Moreover, only predefined sequences are detected [10]. EST sequencing techniques have limitations in the depth of the transcriptome that can be sampled [11].

Recent rapid developments of high-throughput deep sequencing technologies have provided an unprecedented increase in transcriptome data [12]. These nextgeneration sequencing platforms, such as the Solexa/ Illumina Genome Analyzer and ABI/SOLiD Gene Sequencer, can sequence in parallel massive amounts of DNA molecules derived directly from mRNA, producing millions or even billions of high-quality short reads $[13,14]$. DeepSAGE is a tag sequencing method on the Illumina high-throughput sequencing platform that is analogous to LongSAGE $[15,16]$. Compared to LongSAGE, DeepSAGE provides much more sensitive and cost-efficient gene expression profiling $[15,16]$. By using this technology, some progress has recently been made in the characterization of the immune mechanisms and pathways in zebrafish [17]. Nevertheless, there are still important gaps in the knowledge of numerous immune mechanisms, and the available information varies according to the fish species [18].

Here, the large yellow croaker was used as a model to investigate the host response to $A$. hydrophila infection. First, a transcriptome library was constructed from spleen isolated from $A$. hydrophila-infected fish. Deep sequencing was accomplished using the Solexa/Illumina sequencing technology. Using the SOAP de novo transcriptome assembly software, we ultimately obtained a transcriptome database containing 8216 identified unigenes. Quantitative gene expression analysis was performed using DeepSAGE technology. Tags identified from normal and bacteria-infected fish were mapped to the transcriptome database above for comparative analysis. A reference set of significantly upregulated and downregulated immune-related genes was compiled.

\section{Results}

Transcriptome profile of the large yellow croaker (Pseudosciaena crocea)

To better understand the molecular mechanisms of the large yellow croaker immune system, we constructed a Solexa cDNA library from the spleen of fish infected with $A$. hydrophila. High-throughput paired-end sequencing yielded a total of 13,611,340 reads. Of these, 901,200 reads containing more than five consecutive bases with a quality $<13$ were removed. The remaining $12,710,140$ high-quality reads were assembled into 26,313 scaffolds by using the SOAP de novo software, with a maximum scaffold length of $7585 \mathrm{bp}$. The length statistics of all scaffolds are presented in Figure 1.

Scaffold annotation was achieved through BLASTN similarity searches against the zebrafish RefSeq mRNA database (version danRer5). This analysis revealed that 10,502 of the 26,313 scaffolds (40\%) shared homology with zebrafish genes when a cutoff E-value of 1e-05 was used. Scaffolds were clustered if two or more query sequences were annotated to the same zebrafish gene. Ultimately, 5715 unigenes were obtained. Scaffolds that did not display any similarity to zebrafish genes were

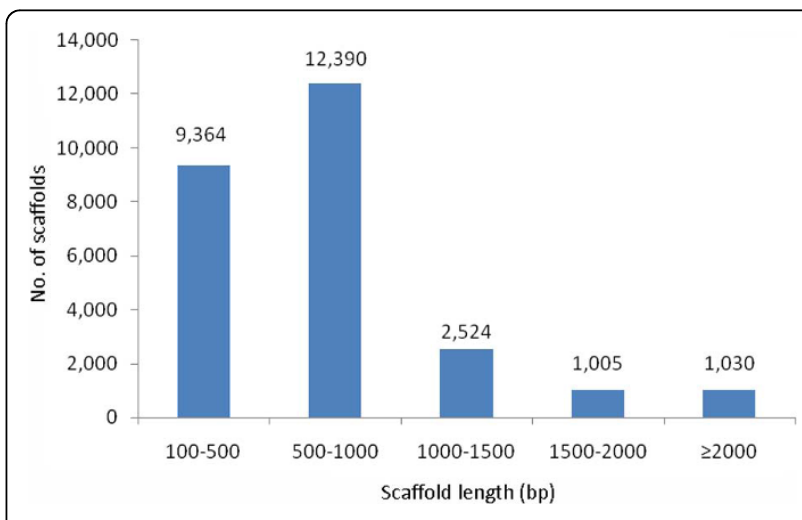

Figure 1 Length statistics of scaffolds obtained from the large yellow croaker transcriptome library. The length distributions of the transcriptome library. Sequences with lengths of 500-1000 bp were most abundant, making up $47 \%$ of the scaffolds. 
further searched against the nonredundant (nr) database, and 2501 unigenes were obtained after clustering. In total, 8216 unigenes were identified in the transcriptome of the large yellow croaker (Additional file 1, Table S1). The remaining 13,102 scaffolds failed to match proteins in the $\mathrm{nr}$ database and therefore represented potentially novel genes.

Gene ontology (GO) analysis of these genes was performed using the web-based Database for Annotation, Visualization, and Integrated Discovery (DAVID) $[19,20]$. Among the 8216 unigenes, DAVID had functional annotation for 5590 genes. The DAVID functional annotation analysis for GO (level 2) is summarized in Table 1. Sequences with GO terms corresponding to the "cellular component" group fell into 14 subcategories, "molecular function" into 16 subcategories, and "biological process" into 31 subcategories. The largest subcategory found in the "cellular component" group was 'cell part,' which comprised $98.8 \%$ of the genes in this subcategory. In the "molecular function" and "biological process" categories, "nucleotide binding" and "primary metabolic process" were the most abundant GO terms, making up $22.4 \%$ and $50.2 \%$ of each subcategory, respectively.

To identify the biological pathways that are active in the large yellow croaker, we mapped the 8216 genes to canonical signaling pathways found in the Kyoto Encyclopedia of Genes and Genomes (KEGG). A total of 3094 genes of the large yellow croaker transcriptome were mapped to KEGG, and 20 statistically remarkable categories $(\mathrm{P}$ value $<0.05)$ are listed in Table 2 . The mitogen-activated protein kinase (MAPK) signaling pathway, neurotrophin signaling pathway, and chemokine signaling pathway were identified as statistically significant. In fact, 47 genes were found to be related to the MAPK pathway. Other major immune pathways, such as those mediated by the $\mathrm{T}$ cell receptor (TCR) and $B$ cell receptor (BCR), were also statistically significant.

\section{Global changes in gene expression upon A. hydrophila infection}

To characterize the immune response of the large yellow croaker to bacterial infection, two DeepSAGE libraries were constructed using mRNA from spleens injected with $A$. hydrophila or $0.9 \% \mathrm{NaCl}$. After removal of the low-quality tags, adaptor tags, and one copynumber tag, a total of 4,841,402 and 5,395,715 clean tags were obtained from the two libraries with 100,107 and 108,572 unique nucleotide sequences, respectively (Additional file 2, Table S2). Subsequently, the tag sequences from the infected and control libraries were mapped to the transcriptome database described above. Approximately $50 \%$ of the tags matched sequences in the transcriptome, while $39 \%$ could be identified unequivocally by unique tag mapping (Additional file 3, Table S3). A total of 1996 differentially expressed genes ( $\mathrm{P}$ value $<0.05$ ) were found (Additional file 4, Table S4), including 1133 upregulated genes and 863 downregulated genes, in the spleen of fish infected with $A$. hydrophila. Particularly, 727 genes were upregulated at least 1.5-fold, including 208 genes that were unique to the infected library, while 489 genes were downregulated at least 1.5 -fold, including 182 genes uniquely expressed in the control library.

To achieve a functional annotation of the infectionresponsive genes, GO classifications were assigned to the 1996 differentially expressed genes by using DAVID (Additional file 5, Table S5). GO analysis indicated that bacterial infection up- and downregulated genes involved in immunity, transcription, translation regulations, and biological regulation.

Some significantly differentially expressed genes in expression profiles using GO classifications are shown in Table 3. The immune-related genes were enriched in GO terms "response to chemical stimulus" and "immune system development." Relative quantitative real-time PCR analysis was also performed to confirm the differentially expression genes. These genes were mapped to KEGG and found to be associated with the Toll-like receptor (TLR) signaling pathway (Figure 2). This group included TLR genes (e.g., TLR1, TLR2, TLR3, and $T L R 22$ ), cytokine genes (e.g., $T N F-\alpha, I L-1 \beta$, and $I L-8)$, and chemokine and chemokine receptor genes (e.g., CCL-4, CCL-c25v, CCR-1, CCR-12.3). Additionally, apoptosis-related genes, including Casp 9 and Fas, as well as those involved in antioxidant activity such as $\operatorname{Prdx1}, \operatorname{Prdx2}, G p x 1 b$, and Gpx4b were discovered. Genes involved in B cell and T cell development, such as $B \operatorname{lnk}$ and $C D 3 \zeta / d$, were also found to be differentially expressed (Table 3 ). The B cell linker protein (Blnk), also known as SLP-65, is essential for normal B cell development by influencing the BCR signaling pathway [21]. The TCR/CD3 $\zeta$ complex mediates antigen recognition and $\mathrm{T}$ cell stimulation, with $\mathrm{CD} 3 \zeta / \mathrm{d}$ playing a pivotal role in this process [22].

Many genes in the transcription regulation group were upregulated by $A$. hydrophila infection. This group includes genes encoding $N F-\kappa B 2, N F-\kappa B i e, I R F 9$, IRF11, Jund, Jak1, Stat1, Cebpa, and Cebpb (Table 3). NF- $\kappa \mathrm{B}$ is a transcription factor involved in regulating a large number of genes, especially cytokine genes [23]. Jak1 and Stat1 are components of the JAK-STAT signaling pathway. The remaining genes were represented by GO terms such as cellular component, binding, catalytic activity, structural molecular activity, and growth. These biological functions and pathways have not been associated directly with a particular immune-related event. 
Table $1 \mathrm{GO}$ function annotation results of 8216 unigenes

\begin{tabular}{|c|c|c|c|c|c|}
\hline Term & GO ID & Description & Gene No. & \%* & $P$ Value \\
\hline$\overline{C C} \_2$ & GO:0044464 & cell part & 2650 & 98.84 & 0.000013 \\
\hline CC_2 & GO:0005622 & intracellular & 2041 & 76.13 & 0.000000 \\
\hline CC_2 & GO:0044424 & intracellular part & 1707 & 63.67 & 0.000000 \\
\hline CC_2 & GO:0043229 & intracellular organelle & 1351 & 50.39 & 0.000000 \\
\hline CC_2 & GO:0043227 & membrane-bounded organelle & 1103 & 41.14 & 0.000000 \\
\hline CC_2 & GO:0044446 & intracellular organelle part & 466 & 17.38 & 0.000000 \\
\hline CC_2 & GO:0044422 & organelle part & 466 & 17.38 & 0.000000 \\
\hline CC_2 & GO:0043234 & protein complex & 410 & 15.29 & 0.000000 \\
\hline CC_2 & GO:0043228 & non-membrane-bounded organelle & 366 & 13.65 & 0.000000 \\
\hline CC_2 & GO:0030529 & ribonucleoprotein complex & 210 & 7.83 & 0.000000 \\
\hline CC_2 & GO:0031090 & organelle membrane & 137 & 5.11 & 0.001153 \\
\hline CC_2 & GO:0043233 & organelle lumen & 135 & 5.04 & 0.000000 \\
\hline CC_2 & GO:0031967 & organelle envelope & 98 & 3.66 & 0.000398 \\
\hline CC_2 & GO:0012505 & endomembrane system & 86 & 3.21 & 0.021749 \\
\hline MF_2 & GO:0000166 & nucleotide binding & 827 & 22.39 & 0.000000 \\
\hline MF_2 & GO:0016787 & hydrolase activity & 674 & 18.25 & 0.000000 \\
\hline MF_2 & GO:0016740 & transferase activity & 606 & 16.4 & 0.000001 \\
\hline MF_2 & GO:0001882 & nucleoside binding & 506 & 13.7 & 0.000000 \\
\hline MF_2 & GO:0016874 & ligase activity & 122 & 3.3 & 0.000001 \\
\hline MF_2 & GO:0003735 & structural constituent of ribosome & 118 & 3.19 & 0.000000 \\
\hline MF_2 & GO:0048037 & cofactor binding & 96 & 2.6 & 0.001411 \\
\hline MF_2 & GO:0060589 & nucleoside-triphosphatase regulator activity & 77 & 2.08 & 0.001763 \\
\hline MF_2 & GO:0008135 & translation factor activity, nucleic acid binding & 60 & 1.62 & 0.000000 \\
\hline MF_2 & GO:0016853 & isomerase activity & 58 & 1.57 & 0.000002 \\
\hline MF_2 & GO:0003702 & RNA polymerase II transcription factor activity & 32 & 0.87 & 0.000002 \\
\hline MF_2 & GO:0051540 & metal cluster binding & 24 & 0.65 & 0.026354 \\
\hline MF_2 & GO:0008430 & selenium binding & 16 & 0.43 & 0.031351 \\
\hline MF_2 & GO:0019825 & oxygen binding & 11 & 0.3 & 0.022460 \\
\hline MF_2 & GO:0004601 & peroxidase activity & 9 & 0.24 & 0.072047 \\
\hline MF_2 & GO:0008641 & small protein activating enzyme activity & 5 & 0.14 & 0.071494 \\
\hline BP_2 & GO:0044238 & primary metabolic process & 1571 & 50.18 & 0.000000 \\
\hline BP_2 & GO:0044237 & cellular metabolic process & 1466 & 46.82 & 0.000000 \\
\hline BP_2 & GO:0043170 & macromolecule metabolic process & 1271 & 40.59 & 0.000000 \\
\hline BP_2 & GO:0009058 & biosynthetic process & 637 & 20.34 & 0.000000 \\
\hline BP_2 & GO:0006807 & nitrogen compound metabolic process & 608 & 19.42 & 0.000020 \\
\hline BP_2 & GO:0051234 & establishment of localization & 533 & 17.02 & 0.051495 \\
\hline BP_2 & GO:0006810 & transport & 528 & 16.86 & 0.055745 \\
\hline BP_2 & GO:0009056 & catabolic process & 235 & 7.51 & 0.000000 \\
\hline BP_2 & GO:0033036 & macromolecule localization & 214 & 6.83 & 0.000000 \\
\hline BP_2 & GO:0045184 & establishment of protein localization & 178 & 5.69 & 0.000000 \\
\hline BP_2 & GO:0006996 & organelle organization & 154 & 4.92 & 0.031676 \\
\hline BP_2 & GO:0051641 & cellular localization & 139 & 4.44 & 0.000000 \\
\hline BP_2 & GO:0051649 & establishment of localization in cell & 132 & 4.22 & 0.000000 \\
\hline BP_2 & GO:0065008 & regulation of biological quality & 124 & 3.96 & 0.055589 \\
\hline BP_2 & GO:0022607 & cellular component assembly & 107 & 3.42 & 0.001415 \\
\hline BP_2 & GO:0042221 & response to chemical stimulus & 88 & 2.81 & 0.067111 \\
\hline BP_2 & GO:0043933 & macromolecular complex subunit organization & 84 & 2.68 & 0.000005 \\
\hline BP_2 & GO:0016192 & vesicle-mediated transport & 75 & 2.4 & 0.000639 \\
\hline BP_2 & GO:0006066 & alcohol metabolic process & 64 & 2.04 & 0.022528 \\
\hline BP_2 & GO:0019725 & cellular homeostasis & 63 & 2.01 & 0.000479 \\
\hline BP_2 & GO:0070271 & protein complex biogenesis & 58 & 1.85 & 0.000005 \\
\hline
\end{tabular}


Table $1 \mathrm{GO}$ function annotation results of $\mathbf{8 2 1 6}$ unigenes (Continued)

\begin{tabular}{|c|c|c|c|c|c|}
\hline BP_2 & GO:0034621 & cellular macromolecular complex subunit organization & 58 & 1.85 & 0.003498 \\
\hline BP_2 & GO:0002520 & immune system development & 54 & 1.72 & 0.000019 \\
\hline BP_2 & GO:0051301 & cell division & 45 & 1.44 & 0.019598 \\
\hline BP_2 & GO:0019637 & organophosphate metabolic process & 38 & 1.21 & 0.029496 \\
\hline BP_2 & GO:0009893 & positive regulation of metabolic process & 37 & 1.18 & 0.060638 \\
\hline BP_2 & GO:0006413 & translational initiation & 25 & 0.8 & 0.000041 \\
\hline BP_2 & GO:0051236 & establishment of RNA localization & 15 & 0.48 & 0.017466 \\
\hline BP_2 & GO:0042440 & pigment metabolic process & 14 & 0.45 & 0.004606 \\
\hline BP_2 & GO:0044087 & regulation of cellular component biogenesis & 13 & 0.42 & 0.008675 \\
\hline
\end{tabular}

*, indicates the percentage of genes in the specific subcategory from each of the three GO ontologies.

Meanwhile, a number of uniquely expressed genes were hypothetical proteins, and future identification of these genes and their function may provide new insights into the immune response to A. hydrophila infection.

\section{GenMAPP analysis reveals genes involved in TCR and MAPK signaling}

To further explore the immune response profiles induced by $A$. hydrophila infection to the level of a single pathway, we performed a map-based pathway analysis by using the GenMAPP software package http:// www.genmapp.org/. In our study, 4004 Mus musculus homologs were used to create the GenMAPP. Mus musculus homologs were identified by searching the 8216 unigenes against the zebrafish RefSeq data downloaded from the UCSC website http://genome.ucsc.edu/ and then the database of HomoloGene at the NCBI http:// www.ncbi.nlm.nih.gov. GenMAPP analysis was performed to identify genes involved in the MAPK pathway (Figure 3). In total, seven genes were identified as highly upregulated upon infection, Casp9, Prkcb1, Hspa5, Radd45a, Dusp7, Rac1, and Casp1. Contrarily, four genes were highly downregulated in response to $A$. hydrophila infection, Map3k12, Crkl, Jun, and Raf1 (Additional file 6, Table S6). We also used GenMAPP to analyze genes involved in TCR signaling. $\mathrm{T}$ cell activation, a key event in adaptive immunity, promotes a variety of signaling cascades that ultimately lead to cytokine production, cell survival, proliferation, and differentiation [24]. The resultant map (Figure 4) revealed eight remarkably downregulated genes (Was, Lyn, Ptpn6, Ctnnb1, Itk, Crkl, Jun, and Ripk2) and seven remarkably upregulated genes (Khdrbs1, Scap2, Vasp, Pik3r2, Cebpb, Zap 70, and $C b l$ ) involved in TCR signaling after $A$. hydrophila infection (Additional file 7, Table S7).

\section{Discussion}

At present, molecular studies on the immune response to pathogens in the large yellow croaker are still rare. To increase our knowledge of host responses to bacterial infection, we firstly analyzed the transcriptome profile of the fish after A. hydrophila infection. Bioinformatic analysis of RNA-seq data should involve mapping of short reads to the genome [17]. However, genome and transcriptome resources for most vertebrate species have not yet been obtained, including the large yellow croaker. We analyzed the transcriptome of the large yellow croaker in advance and obtained a mass of sequence information. Then quantitative gene expression profile analysis was performed, and the tags were mapped to obtained transcriptome database. In the set of highly differentially expressed genes, a number of genes were reported to be involved in immunity and signal transduction, encoding receptors, cytokines, innate defense molecules, enzymes, signal transducers, transcription factors, and other functional proteins.

The innate immune system represents an efficient first line of defense against invading microbial pathogens. TLRs signal the presence of pathogens and elicit an innate immune response. This process has been reported in zebrafish infected with Mycobacterium marinum $[25,26]$. Our data revealed 35 genes involved in TLR cascades in the transcriptome of infected large yellow croaker and 29 differentially expressed genes in expression profiles (Figure 2). TLR1 and TLR2 function together to recognize lipopeptides with a triacylated $\mathrm{N}$-terminal cysteine. TLR1 is only mildly expressed in T. nigroviridis tissues and slightly upregulated in the spleens of LPS-injected fish [27]. Our data demonstrated that TLR1 was upregulated while TLR2 was downregulated at $24 \mathrm{~h}$ after $A$. hydrophila infection (Figure 5A). This result was partly consistent with that reported by Baoprasertkul et al., in which TLR2 expression in the spleens of channel and blue catfish was downregulated initially but upregulated 1 day postinfection with Edwardsiella ictaluri [28]. Bacterial infection has also been shown to induce TLR3 mRNA expression in zebrafish and channel catfish, as well as in channel-blue backcross hybrids following infection with E. tarda and E. ictaluri $[25,29]$. In our study, TLR3 expression was 
Table 2 Statistically significant KEGG classifications of large yellow croaker genes

\begin{tabular}{lccc}
\hline Category & Gene No. & \%* & P value \\
\hline Huntington's disease & 81 & 2.62 & 0.000000 \\
Ribosome & 70 & 2.26 & 0.000000 \\
Pathways in cancer & 70 & 2.26 & 0.000020 \\
Oxidative phosphorylation & 69 & 2.23 & 0.000000 \\
Alzheimer's disease & 67 & 2.17 & 0.000000 \\
Parkinson's disease & 62 & 2 & 0.000000 \\
Ubiquitin mediated proteolysis & 54 & 1.75 & 0.000000 \\
Lysosome & 54 & 1.75 & 0.000000 \\
Purine metabolism & 51 & 1.65 & 0.000192 \\
MAPK signaling pathway & 47 & 1.52 & 0.027690 \\
Regulation of actin cytoskeleton & 47 & 1.52 & 0.000330 \\
Focal adhesion & 43 & 1.39 & 0.000870 \\
Pyrimidine metabolism & 37 & 1.2 & 0.000079 \\
Insulin signaling pathway & 35 & 1.13 & 0.000092 \\
Neurotrophin signaling pathway & 35 & 1.13 & 0.000014 \\
Chemokine signaling pathway & 34 & 1.1 & 0.041450 \\
Proteasome & 32 & 1.03 & 0.000000 \\
T cell receptor signaling pathway & 29 & 0.94 & 0.000161 \\
Leukocyte transendothelial migration & 29 & 0.94 & 0.000788 \\
B cell receptor signaling pathway & 27 & 0.87 & 0.000001 \\
\hline
\end{tabular}

*, indicates the percentage of genes in each pathway from 3094 genes mapped to KEGG.

also upregulated 22.5-fold postinfection (Figure 5A), suggesting that this receptor might be involved in the immune response to bacterial infection in fish in addition to recognizing double-stranded RNA as in mammals. TLR22 is a fish-specific member of this family [30] that has also been found in the large yellow croaker. Recently, TLR22 was found located on the pufferfish cell surface recognizing long dsRNA sequences, whereas mammalian nucleic acid-sensing TLRs are localized in endosomes or the ER of myeloid cells, indicating that TLR22 may be a functional substitute for mammalian TLR3 that monitors for infections by doublestranded RNA viruses [25]. TLR22 was downregulated in the expression profile, implying that TLR22 was suppressed in the early period of $A$. hydrophila infection. Taken together, these results indicate that TLRs are regulated by various components of Gram-negative bacteria, suggesting that multiple TLR-mediated signaling cascades may simultaneously be involved in immune response to bacterial infection.

In our study, $A$. hydrophila infection led to a dramatic increase in the expression of proinflammatory cytokines such as $I L-1 \beta, I L-8$, and TNF- $\alpha$ (Table 3). Studies have reported that these cytokines are induced within $24 \mathrm{~h}$ in human monocytes following Gram-positive and Gramnegative bacterial infection [31]. IL-1 $\beta$ is considered the prototypic multifunctional cytokine that affects nearly all cell types, either alone or in combination with other cytokines response to infection, injury, or immunologic challenge [32]. IL-8 is a proinflammatory CXC chemokine that has been shown to be regulated by a number of different stimuli including inflammatory signals (e.g., $T N F-\alpha, I L-\beta)$, chemical and environmental stresses, and steroid hormones [33]. Here, upregulation of these cytokines was observed by real-time PCR (Figure 5B), which is consistent with the observed findings in DeepSAGE. Therefore, the upregulation of these proinflammatory cytokines strongly suggests that the proinflammatory response may represent an important antibacterial mechanism at the early phase of infection.

The JAK-STAT pathway is initiated in response to cytokines, such as interleukins and IFNs, and growth factors present in the surrounding microenvironment [34]. Jak1 is a cytoplasmic tyrosine kinase that noncovalently associates with a variety of cytokine receptors and plays a nonredundant role in lymphoid cell precursor proliferation, survival, and differentiation $[35,36]$. STAT1, after activation by IFN- $\gamma$ signaling, leads to the activation of peritoneal macrophages, resulting in enhanced bacteria killing and protection against lethal levels of Listeria monocytogenes infection in mice [23]. Genes encoding JAK-STAT pathway members, including Jak1 and Stat1, were found to be upregulated in our study (Table 3), suggesting that the JAK-STAT pathway may be affected by bacterial infection, which may result in changes in other cross-talk biological processes, such as NF- $\kappa$ B signaling pathway, TGF- $\beta$-activated SMAD pathway, and apoptosis [37].

Another signaling pathway affected by bacterial infection in the large yellow croaker was the MAPK cascade. This pathway has been demonstrated to regulate the expression of genes involved in the immune response to pathogens [38], cell differentiation, and cell death [39]. Modulation of MAPK activity in the common periwinkle in response to Escherichia coli-derived LPS has been studied [40]. Some key MAPK-related genes were identified in our transcriptome, including Casp9, Rac1, Gadd45 $\alpha$, and Dusp7 (Additional file 6, Table S6). Quantitative PCR analysis confirmed the differential expression of Casp 9 and Dusp7 (Figure 5C). The Rho family GTPase Rac1 has been implicated in the control of the p38 MAPK signaling pathway by controlling $\beta 1$ integrin. As shown in humans, dominant-negative Rac1 completely inhibits $\beta 1$ integrin-induced p38 MAPK activation, whereas wild-type Rac1 overexpression causes a slight increase in $\beta 1$ integrin-induced p38 MAPK activation [41]. Dual-specificity phosphatases including Dusp 7 are a subset of protein tyrosine phosphatases, many of which dephosphorylate threonine and tyrosine residues on MAPKs and hence are also referred to as MAPK phosphatases (MKPs). The regulated expression and activity of DUSP family members in different cells and 
Table 3 Representative genes significantly differentially expressed after $A$. hydrophila infection

\begin{tabular}{|c|c|c|c|c|}
\hline Gene Name & Accession NO. & Describe & Fold & $P$ value \\
\hline \multicolumn{5}{|c|}{ Immunity related genes } \\
\hline TLR1 & P79800 & Toll-like receptor 1 & $18 / 0$ & 0.000001 \\
\hline TLR2 & NM_212812 & Toll-like receptor 2 & 0/94 & 0 \\
\hline TLR3 & BAD01045 & Toll-like receptor 3 & $4 / 0$ & 0 \\
\hline TLR22 & NM_001128675 & Toll-like receptor 22 & -2.5 & 0 \\
\hline$\| \mathrm{L}-1 \beta$ & gb|AAP33156.1| & Interleukin-1 $\beta$ & +17.9 & 0 \\
\hline IL-8 & XP_695462 & Interleukin-8 & +20.8 & 0.000007 \\
\hline $\mathrm{IL}-2 \mathrm{rgb}$ & NM_001123050 & Interleukin 2 receptor, gamma b & +2.3 & 0.001956 \\
\hline $\mid L-4 r$ & NM_001013282 & Interleukin 4 receptor & +1.4 & 0.000015 \\
\hline IL-6r & NM_001114318 & Interleukin 6 receptor & +2.2 & 0.000023 \\
\hline CCL-4 & CAO78735.1 & CC chemokine ligand 4 & +2.75 & 0 \\
\hline$C C L-C 25 v$ & NM_001115103 & Chemokine CCL-C25V & $0 / 34$ & 0 \\
\hline $\mathrm{CCr}-1$ & ref|NP_001028030.1 & CC chemokine receptor type 1 & +9.6 & 0 \\
\hline $\mathrm{CCr}-12.3$ & NM_001045027 & CC chemokine receptor family-like & +4.4 & 0 \\
\hline Crlf-3 & ref|NP_001167401.1 & cytokine receptor-like factor 3 & $65 / 0$ & 0 \\
\hline TNFaip8 & NM_200332 & TNF, alpha-induced protein 8-like protein 1 & -2.2 & 0.043671 \\
\hline TNFsf10l2 & NM_001002593 & TNF superfamily, member 10 like 2 & -1.3 & 0.00616 \\
\hline Jfmipla & $\mathrm{dbj}|\mathrm{BAC} 10650.1|$ & MIP1alpha & -2.5 & 0.000505 \\
\hline Cklr & gb|AAP58737.1| & C-type lectin receptor & +1.7 & 0 \\
\hline Blnk & NM_212838 & B-cell linker & -1.6 & 0 \\
\hline zgc:55347 & NM_213522 & Immunoglobulin binding protein 1 & $4 / 0$ & 0.026366 \\
\hline Fcgr1 & gb|ACN10126.1| & High affinity immunoglobulin gammaFc receptor I precursor & +2.6 & 0.049003 \\
\hline CD3g/d & ref|NP_001033072.1 & CD3 gamma/delta & -1.9 & 0 \\
\hline $\operatorname{Rad} 23 \mathrm{~b}$ & NM_200564 & RAD23 homolog B & +2.4 & 0.018151 \\
\hline Fas & ref|NP_001075464. & Fas & $4 / 0$ & 0.026366 \\
\hline Casp9 & NM_001007404 & caspase 9 apoptosis-related cysteine protease & +5.8 & 0.000013 \\
\hline Was & emb|CAQ15295.1| & Wiskott-Aldrich syndrome & -3.3 & 0.017699 \\
\hline Tpsn & NM_130974 & Tapasin & +2.3 & 0 \\
\hline Lipf & NM_213404 & Lipf protein & +2.4 & 0 \\
\hline Hsp90a.1 & NM_131328 & Heat shock protein HSP 90-alpha 1 & +1.2 & 0.048885 \\
\hline Gadd45al & NM_200576 & Growth arrest and DNA-damage-inducible, beta & +1.7 & 0 \\
\hline $\operatorname{Prd} \times 1$ & NM_001013471 & Peroxiredoxin 1 & +5.8 & 0 \\
\hline Prd $\times 2$ & NM_001002468 & Peroxiredoxin 2 & -1.5 & 0 \\
\hline Glrx5 & NM_213021 & Glutaredoxin 5 & +1.3 & 0.015063 \\
\hline Gpx1b & NM_001004634 & Glutathione peroxidase $1 \mathrm{~b}$ & +1.4 & 0 \\
\hline Gpx4b & NM_001030070 & Glutathione peroxidase $4 \mathrm{~b}$ & -1.4 & 0 \\
\hline zgc:85657 & NM_214749 & Non-homologous end-joining factor 1 & -1.4 & 0.02618 \\
\hline Mpx & NM_212779 & Myeloid-specific peroxidase & -1.9 & 0.02238 \\
\hline Ube2nl & NM_200342 & Ubiquitin-conjugating enzyme E2N-like & +2 & 0 \\
\hline \multicolumn{5}{|c|}{ Transcription regulator activity } \\
\hline NF-kB2 & NM_001001840 & NF-kB, p49/p100 & -1.7 & 0 \\
\hline NF-kBie & NM_001080089 & NF-kB 2 inhibitor, epsilon & +1.7 & 0 \\
\hline Jak1 & NM_131073 & Janus kinase 1 & +6.1 & 0.003023 \\
\hline Stat1 & ref|NP_001117126.1| & STAT1 alpha & +3.9 & 0.027891 \\
\hline Jun & NM_199987 & c-Jun & $0 / 5$ & 0.02144 \\
\hline Jund & NM_001128342 & Jun D proto-oncogene & +1.5 & 0.012807 \\
\hline Xbp1 & NM_131874 & $\mathrm{X}$-box binding protein 1 & $0 / 6$ & 0.011301 \\
\hline Smad9 & NM_001004014 & Smad9 & -3.6 & 0.031597 \\
\hline Slp-1 & gb|AAC41262.1| & Transcription factor & $0 / 4$ & 0.040678 \\
\hline Srf & gb|AAH50480.1| & Srf protein & -1.5 & 0.006007 \\
\hline Tp53 & NM_131327 & Cellular tumor antigen p53 & -1.2 & 0.031928 \\
\hline
\end{tabular}


Table 3 Representative genes significantly differentially expressed after A. hydrophila infection (Continued)

\begin{tabular}{|c|c|c|c|c|}
\hline Cebpa & NM_131885 & CCAAT/enhancer binding protein alpha & $11 / 0$ & 0.00014 \\
\hline Pdlim1 & NM_001017870 & PDZ and LIM domain 1 & +2.1 & 0.000003 \\
\hline Ahr2b & ref|NP_001033052.1| & Aryl hydrocarbon receptor $2 \mathrm{~B}$ & +2.3 & 0.00573 \\
\hline IRF & dbj|BAA83468.1| & interferon regulatory factor & +2.9 & 0 \\
\hline IRF4 & NM_001122710 & interferon regulatory factor 4 & +1.2 & 0.040115 \\
\hline IRF9 & NM_205710 & interferon regulatory factor 9 & +2.3 & 0 \\
\hline Max & NM_131220 & Myc-associated factor $X$ & +1.3 & 0.003228 \\
\hline Rargb & NM_001083310 & Retinoic acid receptor gamma & $13 / 0$ & 0.000031 \\
\hline Ldb1a & NM_131313 & LIM domain-binding protein 4 & +1.6 & 0.0016 \\
\hline Cse11 & NM_201450 & Chromosome segregation 1-like & -1.2 & 0.045136 \\
\hline Ppp1r10 & NM_212568 & protein phosphatase 1 , regulatory subunit 10 & -1.2 & 0.00723 \\
\hline Ppp1caa & NM_214811 & protein phosphatase 1 , catalytic subunit alpha & -3.9 & 0.007564 \\
\hline Gtf2h2 & NM_201581 & General transcription factor $I I H$, polypeptide 2 & $4 / 0$ & 0.026366 \\
\hline Gtf2h3 & NM_001002564 & General transcription factor $\| \mathrm{H}$, polypeptide 3 & +2.5 & 0.000295 \\
\hline Gf2f2 & NM_001017832 & General transcription factor IIF, polypeptide 2 & +1.4 & 0.021645 \\
\hline Gtf2e2 & NM_212731 & General transcription factor IIE, polypeptide 2, beta & +31.9 & 0 \\
\hline
\end{tabular}

Limitations of all differentially expressed genes are based on $\mathrm{P}<0.05$. A P value $<0.05$ indicated that the gene was significantly altered after bacterial challenge. The absolute value of "Fold" means the magnitude of up- or downregulation for each gene/homolog after bacterial challenge; "+" indicates upregulation, "-" indicates downregulation, and " 0 " indicates the gene was not found in one library. "Accession NO" is GenBank identifiers for the conformable reference sequences.

tissues control MAPK intensity and duration to determine the type of physiological response [42,43]. Therefore, the identified changes in gene expression in the large yellow croaker may facilitate the activation of the MAPK pathway and protect hosts against $A$. hydrophila infection.
Adaptive immunity is the process that leads to specific host resistance to infection [44]. T cells orchestrate responses against such foreign pathogens as viruses and bacteria. TCR and its downstream signaling cascades play a key role in these events. Here, we identified TCR pathway-related genes that were downregulated at $24 \mathrm{~h}$

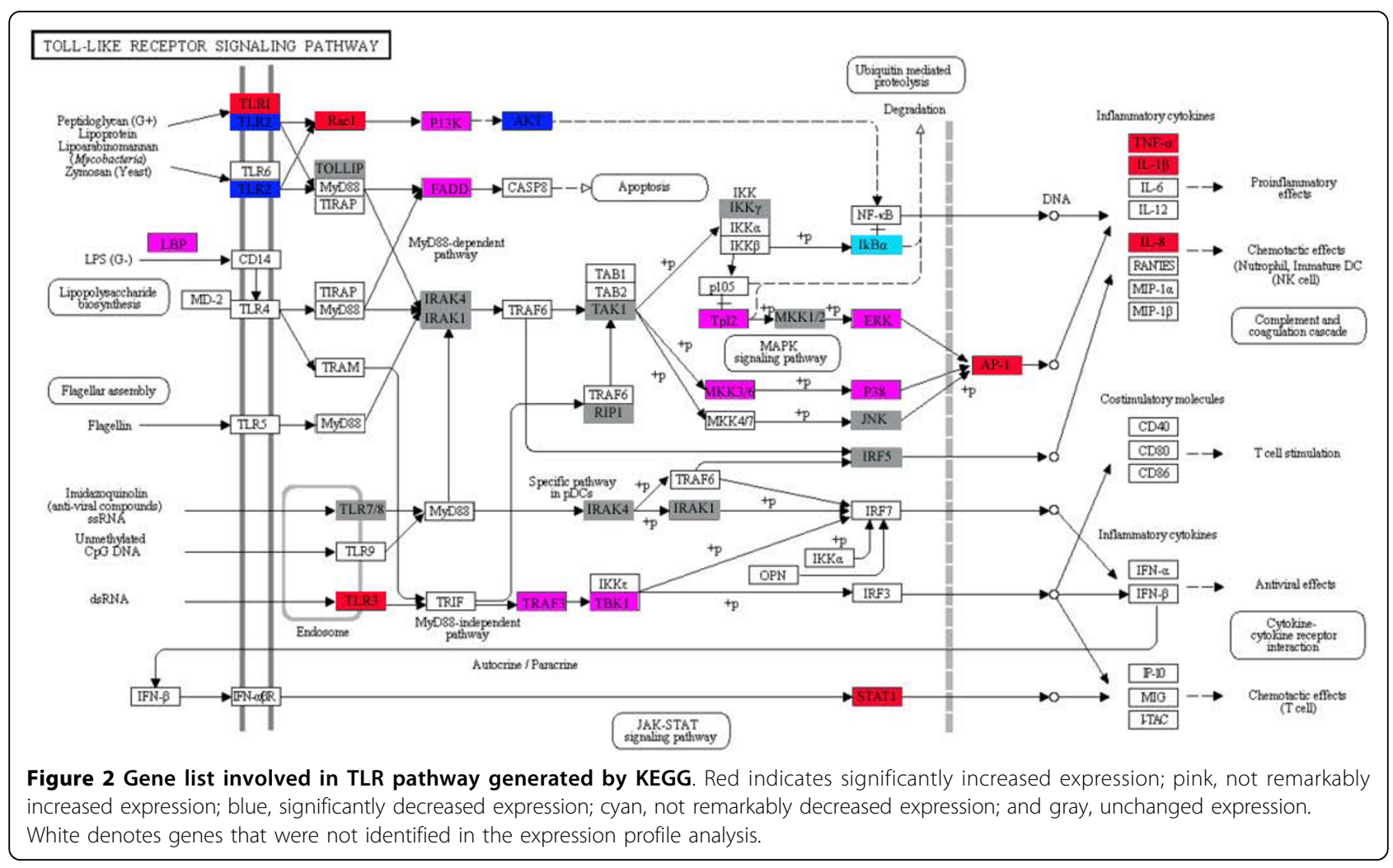




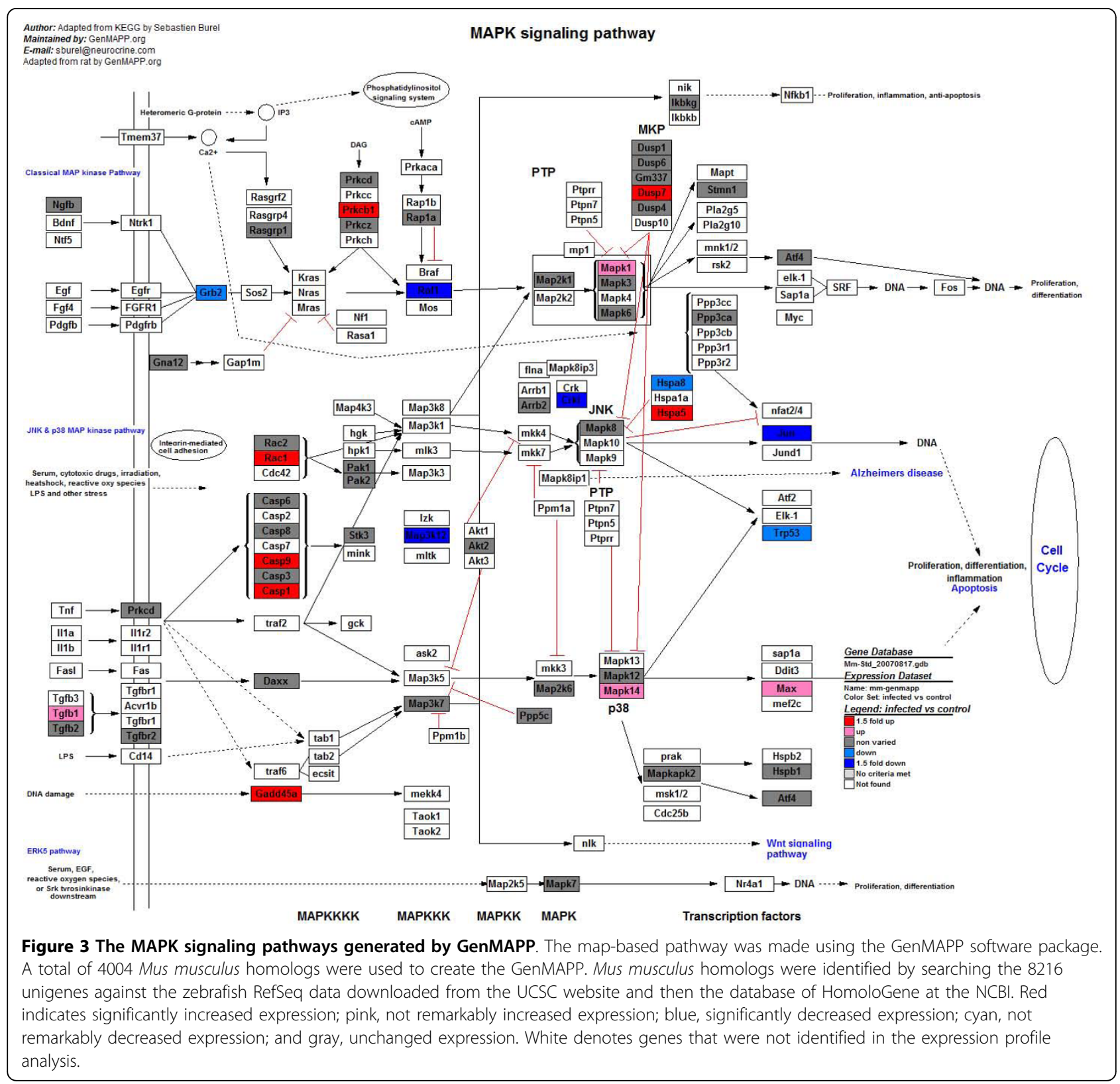

after $A$. hydrophila infection. This complex process is shown in Figure 4, and genes expressed differentially are listed in Additional file 7, Table S7. Lyn, Itk, Was, Ptpn6, and Jun expression was downregulated, implying that the TCR signaling pathway may be suppressed in the early period $(24 \mathrm{~h})$ following bacterial infection. Studies have shown that a fine balance exists between a positive signal that initiates TCR cascade and a negative signal that controls the threshold, extent, and termination of TCR activation [45]. Several protein tyrosine phosphatases (PTPs) have been shown to function as negative regulators of the TCR signaling pathway by dephosphorylating activated signaling molecules $[46,47]$.
Here, expression of Ptpn6, a member of the PTP family [48], was downregulated (Figure 5D), suggesting that although the TCR signaling pathway was suppressed by A. hydrophila, the host began to downregulate the expression of the PTPs to antagonize the repression. Clearly, there is a need for further studies to elucidate the precise roles of the PTP family members in the TCR signaling pathway in fish.

\section{Conclusions}

Several recent studies have exploited novel highthroughput deep sequencing technology as a new method to advance further understanding of the 


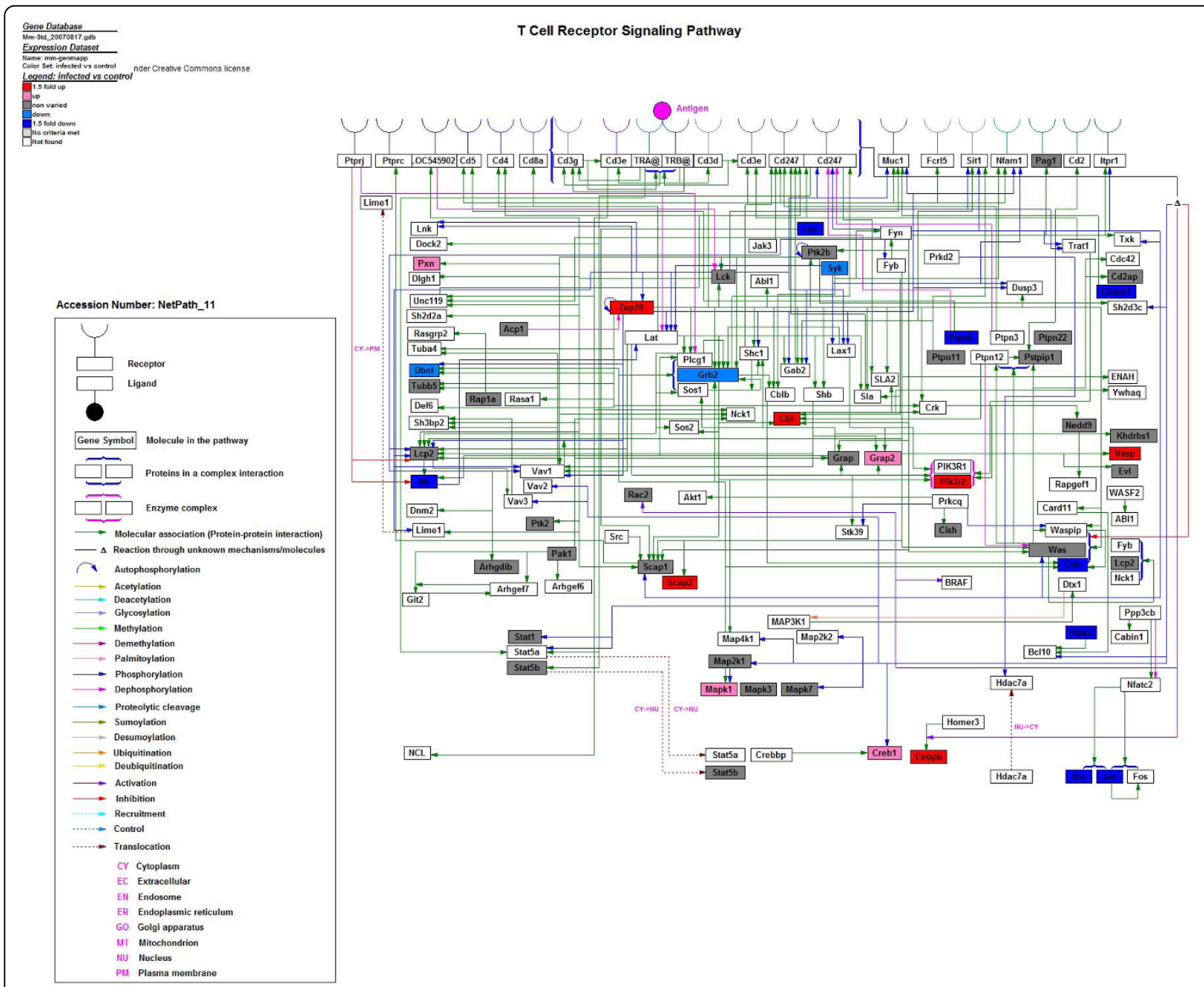

Figure 4 The TCR signaling pathways generated by GenMAPP. The map-based pathway was made using the GenMAPP software package. A total of 4004 Mus musculus homologs were used to create the GenMAPP. Mus musculus homologs were identified by searching the 8216 unigenes against the zebrafish RefSeq data downloaded from the UCSC website and then the database of HomoloGene at the NCBI. Red indicates significantly increased expression; pink, not remarkably increased expression; blue, significantly decreased expression; cyan, not remarkably decreased expression; and gray, unchanged expression. White denotes genes that were not identified in the expression profile analysis.

mechanism of fish defense against infection [17]. We used the A. hydrophila-infected large yellow croaker as a model to study the immune response of fish to bacterial infection. Our analysis of the transcriptome and gene expression in A. hydrophila-infected large yellow croaker revealed changes in multiple signaling pathways involved in immunity in the large yellow croaker. The multiple TLR-mediated signaling cascades may be involved in early response to bacterial infection, causing the production of proinflammatory cytokines, chemokines, and other cytokines, which may result in the inflammatory response and affect other signal pathways such as JAK-STAT and MAPK. However, the TCR signaling pathway, a pivotal process in cellular immunity, was suppressed in the early period of A. hydrophila infection. The immune-related genes and signaling pathways involved in bacterial infection were identified and thereby provided valuable leads for further investigations into the immune response of fish.

\section{Methods}

\section{Fish and infection experiments}

Large yellow croakers (mean weight, $200 \mathrm{~g}$ ) were purchased from a mariculture farm in Lianjian, Fuzhou, China. The fish were maintained at $25^{\circ} \mathrm{C}$ in aerated water tanks with a flow-through seawater supply. After 7 days of acclimation, these fish were used for the infection experiments. Twenty fish were injected intramuscularly 

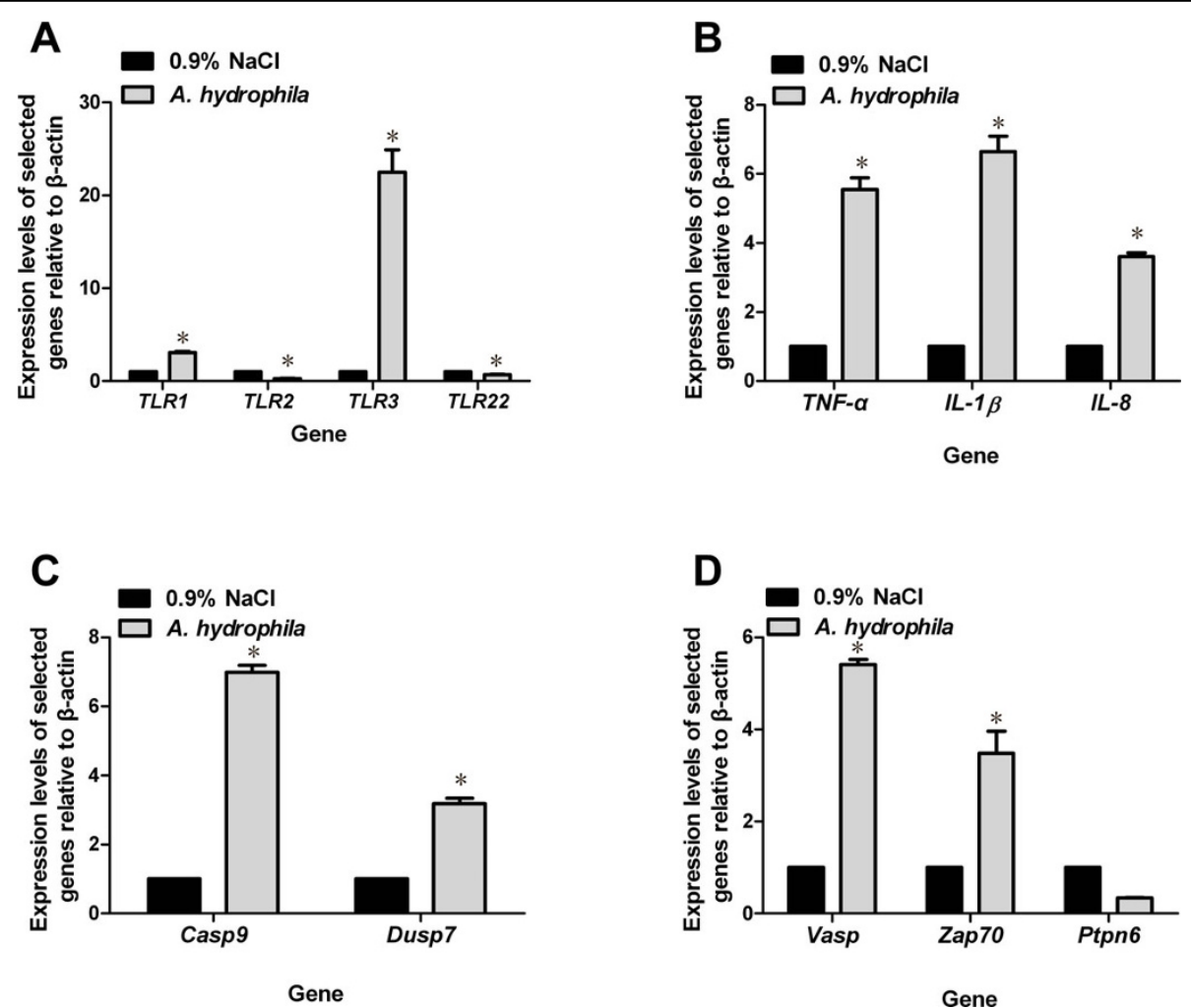

Figure 5 The expression analysis of selected genes from the expression profile by relative quantitative real-time PCR. Total RNA was extracted from spleens of fish infected with $\mathrm{A}$. hydrophila or injected with $0.9 \% \mathrm{NaCl}$. Real-time PCR was used to validate gene expression changes in the TLR pathway (A), cytokines (B), the MAPK signaling pathway (C), and the TCR signaling pathway (D). Increases and decreases in relative levels of transcripts with respect to the control $\beta$-actin gene are shown. For each gene, the black bar indicates the gene expression ratio of fish injected with $0.9 \% \mathrm{NaCl}$ and is defined as 1 ; the grey bar indicates the expression ratio of fish infected with $A$. hydrophila, with associated standard error bars. Statistical significance of the relative expression ratio is indicated (*, $\mathrm{P}<0.01)$.

with $A$. hydrophila at a dose of $1 \times 10^{8} \mathrm{cfu} / 200 \mathrm{~g}$ (This dose was chosen based on previous unpublished data) of fish. The strain of A. hydrophila (PPD 134/91) used in our manuscript was kindly provided by professor Xuanxian Peng [49]. A second group of 20 fish was injected with sterilized $0.9 \% \mathrm{NaCl}$ at a dose of $0.2 \mathrm{ml} / 200 \mathrm{~g}$ of fish as a control [50]. The spleen tissues sampled at $12 \mathrm{~h}$ after infection with $A$. hydrophila were used for transcriptome analysis. The spleen tissues sampled at $24 \mathrm{~h}$ after injections with $A$. hydrophila or $0.9 \% \mathrm{NaCl}$ were used for gene expression profiling analysis. All experiments were conducted in Third Institute of Oceanography, SOA, China. The protocols used meet the "Regulations for the Administration of Affairs Concerning Experimental Animals" established by the Fujian Provincial Department of Science and Technology on the Use and Care of Animals.

\section{RNA isolation}

Total RNA was extracted from 50 to $100 \mathrm{mg}$ of tissue with $\mathrm{TRIZOL}^{\circ}$ Reagent (Invitrogen, Carlsbad, CA, USA) according to the manufacturer's instructions. The RNA samples were incubated for $30 \mathrm{~min}$ at $37^{\circ} \mathrm{C}$ with 10 units of DNaseI (Takara, Dalian, China) to remove residual genomic DNA. The quality and quantity of the purified RNA were determined by measuring the absorbance at $260 \mathrm{~nm} / 280 \mathrm{~nm}$ (A260/A280) using a Nanodrop ND-1000 spectrophotometer (LabTech, Holliston, MA, USA). The samples had an average RIN value of 8.9 according to Labon-chip analysis using the 2100 Bioanalyzer (Agilent Technologies, Santa Clara, CA, USA).

\section{Library preparation and sequencing}

First, to survey the gene expression profile in the large yellow croaker and obtain longer transcript sequences for better annotation of the transcriptome, we constructed the entire library using the Mate Pair Library Preparation Kit. Then, to investigate the dynamics of gene expression after infection with $A$. hydrophila, we performed two tag-library preparations using the DeepSAGE: Tag Profiling for Nla III Sample Prep Kit 
from Illumina according to the manufacturer's instructions.

To better assemble the entire transcriptome de novo, a paired-end sequencing strategy was used for sequencing. A fragment sequencing strategy was used to sequence the tags. The data has been submitted to NCBI, and the accession number is SRA010789.13.

\section{Assembly of transcripts and annotation}

Transcripts were assembled using the SOAP de novo software http://soap.genomics.org.cn/soapdenovo.html. As a result, 26,313 scaffolds were generated. To annotate these scaffolds, we first aligned them by using the zebrafish RefSeq mRNA database. The remaining nonannotated scaffolds were further aligned to the $\mathrm{nr}$ database. The annotated scaffolds were clustered and designated as unigenes when two or more query sequences were annotated to the same gene. The assembled contigs were used as a reference for annotating the DeepSAGE tags. GO and KEGG gene function were performed using DAVID [19].

\section{Identification of differentially expressed genes}

Gene expression was measured by counting tags from normal and bacteria-infected fish and normalized to the total high-quality reads. High-throughput sequencing was performed using the Solexa/Illumina Genome Analyzer. To investigate differences in gene expression profiles, we analyzed genes between both libraries using the IDEG6 modeling methods [51]. GenMAPP 2.0 was used to show differences in expression in the different pathways [52].

\section{Quantitative real-time PCR}

Quantitative real-time PCR was performed using the ABI Prism 7500 Detection System (Applied Biosystems, Foster City, CA, USA) with SYBR Green as the fluorescent dye according to the manufacturer's protocol (Takara). First-strand cDNA was synthesized from $2 \mu \mathrm{g}$ of total RNA as described above and used as a template for real-time PCR with specific primers (Additional file 8, Table S8). Real-time PCR was performed in a total volume of $20 \mu \mathrm{l}$, and cycling conditions were $95^{\circ} \mathrm{C}$ for $5 \mathrm{~min}$, followed by 40 cycles of $94^{\circ}$ $\mathrm{C}$ for $5 \mathrm{~s}, 55^{\circ} \mathrm{C}$ for $20 \mathrm{~s}$, and $72^{\circ} \mathrm{C}$ for $20 \mathrm{~s}$. All reactions were performed in biological triplicates, and the results were expressed relative to the expression levels of $\beta$-actin in each sample by using the $2 \Delta \Delta C T$ method [53]. Each sample was first normalized for the amount of template added by comparison with the abundance of $\beta$-actin mRNA [54].

\section{Additional material}

Additional file 1: Table S1: Details on 8216 unigenes identified in the transcriptome of the large yellow croaker.

Additional file 2: Table S2: Solexa tag libraries of the infected and normal large yellow croaker.

Additional file 3: Table S3: Tags found to match sequences in the transcriptome.

Additional file 4: Table S4: Details on 1996 differentially expressed genes in expression profile of large yellow croaker. The data show the 1996 unigenes that were differentially expressed in the infected and normal large yellow croaker. The tag number, fold change, and $P$ value are shown in the table.

Additional file 5: Table S5: GO function annotation results of 1996 differentially expressed genes using DAVID. Gene Ontology was performed using DAVID.

Additional file 6: Table S6: Significant differentially expressed genes in MAPK signaling pathway.

Additional file 7: Table S7: Significant differentially expressed genes in $\mathrm{T}$ cell receptor signaling pathway.

Additional file 8: Table S8: Primers for relative quantitative realtime PCR. Primers were designed from the sequences of the large yellow croaker transcriptome library by using Primer Premier 5.0.

\section{Acknowledgements}

The work was supported by grants from the Nation ' 863 ' Project (2006AA10A402 and 2007AA091406) and National Natural Science Foundation of China (30871925 and 31001131).

\section{Author details}

${ }^{1}$ Key Laboratory of Marine Biogenetic Resources, Third Institute of Oceanography, State Oceanic Administration, Xiamen 361005, China. ${ }^{2}$ The CAS Key Laboratory of Genome Sciences and Information, Beijing Institute of Genomics, Chinese Academy of Sciences, Beijing 100029, China.

\section{Authors' contributions}

JQA, SNH, and XHC participated in designing the research and helped write the manuscript. YNM performed the research. FD and PC analyzed the data and designed the tables and figures. YNM and FD wrote the manuscript. All authors read and approved the final manuscript.

Received: 13 May 2010 Accepted: 22 September 2010

Published: 22 September 2010

\section{References}

1. Wu ZP, Wang SY: Experimental Study on Immune Protection of Trivalent Vaccine to Common Bacterial Diseases of Pseudosciaena crocea. J Xiamen Univ (Natural Sci) 2004, 43:115-118.

2. Zheng W, Liu G, Ao J, Chen X: Expression analysis of immune-relevant genes in the spleen of large yellow croaker (Pseudosciaena crocea) stimulated with poly I:C. Fish Shellfish Immunol 2006, 21(4):414-430.

3. Cao D, Kocabas A, Ju Z, Karsi A, Li P, Patterson A, Liu Z: Transcriptome of channel catfish (Ictalurus punctatus): initial analysis of genes and expression profiles of the head kidney. Anim Genet 2001, 32(4):169-188.

4. Martin SA, Caplice NC, Davey GC, Powell R: EST-based identification of genes expressed in the liver of adult Atlantic salmon (Salmo salar). Biochem Biophys Res Commun 2002, 293(1):578-585.

5. Shiue YL, Wang LH, Chao TY, Lin CH, Tsai CL: EST-based identification of genes expressed in the hypothalamus of adult tilapia, Oreochromis mossambicus. Biochem Biophys Res Commun 2004, 316(2):523-527.

6. Meijer AH, Verbeek FJ, Salas-Vidal E, Corredor-Adamez M, Bussman J, van der Sar AM, Otto GW, Geisler R, Spaink HP: Transcriptome profiling of 
adult zebrafish at the late stage of chronic tuberculosis due to Mycobacterium marinum infection. Mol Immunol 2005, 42(10):1185-1203.

7. Bayne CJ, Gerwick L, Wheeler PA, Thorgaard GH: Transcriptome profiles of livers and kidneys from three rainbow trout (Oncorhynchus mykiss) clonal lines distinguish stocks from three allopatric populations. Comparative Biochemistry and Physiology 2006, 1:396-403.

8. Peatman E, Terhune J, Baoprasertkul P, Xu P, Nandi S, Wang S, Somridhivej B, Kucuktas H, Li P, Dunham R, et al: Microarray analysis of gene expression in the blue catfish liver reveals early activation of the MHC class I pathway after infection with Edwardsiella ictaluri. Mol Immunol 2008, 45(2):553-566

9. Boswell MG, Wells MC, Kirk LM, Ju Z, Zhang Z, Booth RE, Walter RB: Comparison of gene expression responses to hypoxia in viviparous (Xiphophorus) and oviparous (Oryzias) fishes using a medaka microarray. Comp Biochem Physiol C Toxicol Pharmacol 2009, 149(2):258-265.

10. $\mathrm{t}$ Hoen PA, Ariyurek $\mathrm{Y}$, Thygesen $\mathrm{HH}$, Vreugdenhil E, Vossen $\mathrm{RH}$, de Menezes RX, Boer JM, van Ommen GJ, den Dunnen JT: Deep sequencingbased expression analysis shows major advances in robustness, resolution and inter-lab portability over five microarray platforms. Nucleic Acids Res 2008, 36(21):e141.

11. Hanriot L, Keime C, Gay N, Faure C, Dossat C, Wincker P, Scote-Blachon C, Peyron C, Gandrillon O: A combination of LongSAGE with Solexa sequencing is well suited to explore the depth and the complexity of transcriptome. BMC Genomics 2008, 9:418.

12. Han X, Wu X, Chung WY, Li T, Nekrutenko A, Altman NS, Chen G, Ma H: Transcriptome of embryonic and neonatal mouse cortex by highthroughput RNA sequencing. Proc Natl Acad Sci USA 2009, 106(31):12741-12746

13. Morozova O, Hirst M, Marra MA: Applications of new sequencing technologies for transcriptome analysis. Annu Rev Genomics Hum Genet 2009, 10:135-151.

14. Morozova O, Marra MA: Applications of next-generation sequencing technologies in functional genomics. Genomics 2008, 92(5):255-264.

15. Nielsen KL, Hogh AL, Emmersen J: DeepSAGE-digital transcriptomics with high sensitivity, simple experimental protocol and multiplexing of samples. Nucleic Acids Res 2006, 34(19):e133.

16. Morrissy AS, Morin RD, Delaney A, Zeng T, McDonald $H$, Jones $S$, Zhao $Y$, Hirst M, Marra MA: Next-generation tag sequencing for cancer gene expression profiling. Genome Res 2009, 19(10):1825-1835.

17. Hegedus Z, Zakrzewska A, Agoston VC, Ordas A, Racz P, Mink M, Spaink HP, Meijer AH: Deep sequencing of the zebrafish transcriptome response to mycobacterium infection. Mol Immunol 2009, 46(15):2918-2930.

18. Alvarez-Pellitero P: Fish immunity and parasite infections: from innate immunity to immunoprophylactic prospects. Vet Immunol Immunopathol 2008, 126(3-4):171-198.

19. Huang da W, Sherman BT, Lempicki RA: Systematic and integrative analysis of large gene lists using DAVID bioinformatics resources. Nat Protoc 2009, 4(1):44-57.

20. Huang da W, Sherman BT, Tan Q, Kir J, Liu D, Bryant D, Guo Y, Stephens R, Baseler MW, Lane HC, et al: DAVID Bioinformatics Resources: expanded annotation database and novel algorithms to better extract biology from large gene lists. Nucleic Acids Res 2007, , 35 Web Server: W169-175.

21. Kelly ME, Chan AC: Regulation of B cell function by linker proteins. Curr Opin Immunol 2000, 12(3):267-275.

22. Januchowski $R$, Jagodzinski PP: Effect of 5-azacytidine and procainamide on CD3-zeta chain expression in Jurkat T cells. Biomed Pharmacother 2005, 59(3):122-126.

23. Schindler C, Plumlee C: Inteferons pen the JAK-STAT pathway. Semin Cell Dev Biol 2008, 19(4):311-318.

24. Smith-Garvin JE, Koretzky GA, Jordan MS: T cell activation. Annu Rev Immunol 2009, 27:591-619.

25. Rebl A, Goldammer T, Seyfert HM: Toll-like receptor signaling in bony fish. Vet Immunol Immunopathol 2010, 134(3-4):139-50.

26. Lin $B$, Chen $S$, Cao Z, Lin Y, Mo D, Zhang H, Gu J, Dong M, Liu Z, Xu A: Acute phase response in zebrafish upon Aeromonas salmonicida and Staphylococcus aureus infection: striking similarities and obvious differences with mammals. Mol Immunol 2007, 44(4):295-301.

27. Chang MX, Nie P: RNAi suppression of zebrafish peptidoglycan recognition protein 6 (zfPGRP6) mediated differentially expressed genes involved in Toll-like receptor signaling pathway and caused increased susceptibility to Flavobacterium columnare. Vet Immunol Immunopathol 2008, 124(3-4):295-301.

28. Baoprasertkul P, Peatman E, Abernathy J, Liu Z: Structural characterisation, expression analysis of Toll-like receptor 2 gene from catfish. Fish Shellfish Immunol 2007, 22:418-426.

29. Bilodeau AL, Waldbieser GC: Activation of TLR3 and TLR5 in channel catfish exposed to virulent Edwardsiella ictaluri. Dev Comp Immunol 2005, 29(8):713-721.

30. Jault C, Pichon L, Chluba J: Toll-like receptor gene family and TIR-domain adapters in Danio rerio. Mol Immunol 2004, 40(11):759-771.

31. Hessle CC, Andersson B, Wold AE: Gram-positive and Gram-negative bacteria elicit different patterns of pro-inflammatory cytokines in human monocytes. Cytokine 2005, 30(6):311-318.

32. Church LD, Cook GP, McDermott MF: Primer: inflammasomes and interleukin 1 beta in inflammatory disorders. Nat Clin Pract Rheumatol 2008, 4(1):34-42.

33. Waugh DJ, Wilson C: The interleukin-8 pathway in cancer. Clin Cancer Res 2008, 14(21):6735-6741

34. Pear WS, Aster JC: T cell acute lymphoblastic leukemia/lymphoma: a human cancer commonly associated with aberrant NOTCH1 signaling. Curr Opin Hematol 2004, 11(6):426-433.

35. Haan C, Is'harc H, Hermanns HM, Schmitz-Van De Leur H, Kerr IM, Heinrich PC, Grotzinger J, Behrmann I: Mapping of a region within the N terminus of Jak1 involved in cytokine receptor interaction. J Biol Chem 2001, 276(40):37451-37458

36. Flex E, Petrangeli V, Stella L, Chiaretti S, Hornakova T, Knoops L, Ariola C, Fodale V, Clappier E, Paoloni F, et al: Somatically acquired JAK1 mutations in adult acute lymphoblastic leukemia. J Exp Med 2008, 205(4):751-758.

37. Shuai K, Liu B: Regulation of JAK-STAT signalling in the immune system. Nat Rev Immunol 2003, 3(11):900-911.

38. Tarrega C, Pulido R: A one-step method to identify MAP kinase residues involved in inactivation by tyrosine- and dual-specificity protein phosphatases. Anal Biochem 2009, 394(1):81-86.

39. Hrstka R, Stulik J, Vojtesek B: The role of MAPK signal pathways during Francisella tularensis LVS infection-induced apoptosis in murine macrophages. Microbes Infect 2005, 7(4):619-625.

40. lakovleva NV, Gorbushin AM, Storey KB: Modulation of mitogen-activated protein kinases (MAPK) activity in response to different immune stimuli in haemocytes of the common periwinkle Littorina littorea. Fish Shellfish Immunol 2006, 21(3):315-324.

41. Mainiero F, Soriani A, Strippoli R, Jacobelli J, Gismondi A, Piccoli M, Frati L, Santoni A: RAC1/P38 MAPK signaling pathway controls beta1 integrininduced interleukin-8 production in human natural killer cells. Immunity 2000, 12(1):7-16.

42. Jeffrey KL, Camps M, Rommel C, Mackay CR: Targeting dual-specificity phosphatases: manipulating MAP kinase signalling and immune responses. Nat Rev Drug Discov 2007, 6(5):391-403.

43. Owens DM, Keyse SM: Differential regulation of MAP kinase signalling by dual-specificity protein phosphatases. Oncogene 2007, 26(22):3203-3213.

44. Orme I: Adaptive immunity to mycobacteria. Curr Opin Microbiol 2004, 7(1):58-61.

45. Qian D, Weiss A: T cell antigen receptor signal transduction. Curr Opin Cell Biol 1997, 9(2):205-212.

46. Mustelin T, Rahmouni S, Bottini N, Alonso A: Role of protein tyrosine phosphatases in T cell activation. Immunol Rev 2003, 191:139-147.

47. Mohi MG, Neel BG: The role of Shp2 (PTPN11) in cancer. Curr Opin Genet Dev 2007, 17(1):23-30.

48. Alonso A, Sasin J, Bottini N, Friedberg I, Osterman A, Godzik A, Hunter T, Dixon J, Mustelin T: Protein tyrosine phosphatases in the human genome. Cell 2004, 117(6):699-711.

49. Peng X, Zhang J, Wang S, Lin Z, Zhang W: Immuno-capture PCR for detection of Aeromonas hydrophila. J Microbiol Methods 2002, 49(3):335-338.

50. Wan X, Chen X: Molecular cloning and expression analysis of a CXC chemokine gene from large yellow croaker Pseudosciaena crocea. Vet Immunol Immunopathol 2009, 127(1-2):156-161.

51. Romualdi C, Bortoluzzi S, D'Alessi F, Danieli GA: IDEG6: a web tool for detection of differentially expressed genes in multiple tag sampling experiments. Physiol Genomics 2003, 12(2):159-162. 
52. Salomonis N, Hanspers K, Zambon AC, Vranizan K, Lawlor SC, Dahlquist KD, Doniger SW, Stuart J, Conklin BR, Pico AR: GenMAPP 2: new features and resources for pathway analysis. BMC Bioinformatics 2007, 8:217.

53. Livak KJ, Schmittgen TD: Analysis of relative gene expression data using real-time quantitative PCR and the 2(-Delta Delta $C(T)$ ) Method. Methods 2001, 25(4):402-408.

54. Yu S, Ao J, Chen X: Molecular characterization and expression analysis of MHC class II alpha and beta genes in large yellow croaker (Pseudosciaena crocea). Mol Biol Rep 2010, 37(3):1295-1307.

doi:10.1186/1471-2164-11-506

Cite this article as: Mu et al:: Transcriptome and expression profiling analysis revealed changes of multiple signaling pathways involved in immunity in the large yellow croaker during Aeromonas hydrophila infection. BMC Genomics 2010 11:506.

Submit your next manuscript to BioMed Central and take full advantage of:

- Convenient online submission

- Thorough peer review

- No space constraints or color figure charges

- Immediate publication on acceptance

- Inclusion in PubMed, CAS, Scopus and Google Scholar

- Research which is freely available for redistribution

Submit your manuscript at www.biomedcentral.com/submit 